\title{
Clinical Features and Association with Visceral Malignancy in 80 Patients with Sebaceous Neoplasms
}

\author{
Jee Eun Kim, Jong Hoon Kim, Kee-Yang Chung ${ }^{1}$, Jin Sook Yoon², Mi Ryung Roh \\ Department of Dermatology, Gangnam Severance Hospital, Cutaneous Biology Research Institute, Yonsei University College of Medicine, \\ ${ }^{1}$ Department of Dermatology, Severance Hospital, Cutaneous Biology Research Institute, Yonsei University College of Medicine, ${ }^{2}$ Institute of \\ Vision Research, Department of Ophthalmology, Yonsei University College of Medicine, Seoul, Korea
}

Background: Sebaceous neoplasm is a rare subgroup of appendageal tumors that differentiate towards sebaceous adnexal structures of the skin and are known to be associated with visceral malignancy. Objective: We aimed to analyze and compare the clinical data including the past history of associated visceral malignancy in patients with sebaceous carcinoma (SC) and benign sebaceous neoplasms (BSN), such as sebaceous adenomas and sebaceomas. Methods: We retrospectively reviewed the cases of consecutive patients diagnosed with sebaceous neoplasms. Basic demographic data, past medical history, and clinical data regarding the size, location, and presence of associated visceral malignancies were evaluated. Results: A total of 80 patients of sebaceous neoplasms ( 51 SC, 29 BSN) were included. A total of 18 associated visceral malignancies were found in 14 patients (8 SC, $6 \mathrm{BSN})$. Two patients were diagnosed with subsequent visceral malignancies during the primary work-up process for sebaceous neoplasms. The mean age at diagnosis of the visceral malignancies was 63.9 and 47.5 years for patients with SC and BSN, respectively. The most common site of visceral malignancies was the gastrointestinal (Gl) tract. Adenocarcinoma was the most common histologic type of the visceral

Received May 11, 2018, Revised July 25, 2018, Accepted for publication July 27, 2018

Corresponding author: Mi Ryung Roh, Department of Dermatology, Gangnam Severance Hospital, 211 Eonju-ro, Gangnam-gu, Seoul 06273, Korea. Tel: 82-2-2019-3472, Fax: 82-2-3463-6136, E-mail: karenroh@ yuhs.ac

ORCID: https://orcid.org/0000-0002-6285-2490

This is an Open Access article distributed under the terms of the Creative Commons Attribution Non-Commercial License (http://creativecommons. org/licenses/by-nc/4.0) which permits unrestricted non-commercial use, distribution, and reproduction in any medium, provided the original work is properly cited.

Copyright (c) The Korean Dermatological Association and The Korean Society for Investigative Dermatology malignancy noted. Conclusion: We observed associated visceral malignancies in $15.7 \%$ of patients with SC and $20.7 \%$ with BSN. Our results suggest a need for screening of visceral malignancies, especially of the Gl tract, in patients with sebaceous neoplasms. (Ann Dermatol 31(1) 14 21, 2019)

\section{-Keywords-}

Sebaceoma, Sebaceous adenoma, Sebaceous carcinoma, Visceral malignancy

\section{INTRODUCTION}

Sebaceous neoplasm is a rare subgroup of appendageal tumor that differentiates towards sebaceous adnexal structures of the skin. Appendageal tumors with sebaceous differentiation are widely classified into 3 subgroups according to the gradient of decreasing differentiation: (1) hyperplasias and hamartomas - nevus sebaceous and sebaceous hyperplasia, (2) benign neolplasms - sebaceous adenoma and sebaceoma and (3) malignant neoplasms - sebaceous carcinoma (SC) ${ }^{1}$. Sebaceous neoplasms may occur at any age but usually affect elderly population. These tumors most commonly present on the head and neck, and due to their heterogeneous clinical features, are often difficult to distinguish from other cutaneous neoplasms such as basal cell carcinoma and squamous cell carcinoma. Therefore, histopathologic examination is required for definitive diagnosis ${ }^{2}$. Despite advances in understanding the histopathologic features of sebaceous neoplasms, relatively little is known about the clinical features of patients with sebaceous neoplasms, especially in those with benign sebaceous neoplasm (BSN). Additionally, sebaceous adenomas are known to be associated with visceral malignancy ${ }^{1}$, However, there are few published studies assessing the 
clinical features of associated visceral malignancy such as the frequency and location in the patients with Muir-Torre syndrome (MTS) and there are no previous studies assessing associated visceral malignancy in patients with sebaceous neoplasm without MTS. Information regarding associated visceral malignancy in patients with sebaceous neoplasm would be helpful in the diagnosis of visceral malignancy considering that sebaceous neoplasms are visible lesions which can be easily diagnosed with skin biopsy.

Therefore, in this study we examined the basic demographic data, presenting clinical features and characteristics of associated visceral malignancies in Korean patients diagnosed with sebaceous neoplasms.

\section{MATERIALS AND METHODS}

\section{Study subjects}

This retrospective case review was performed at two tertiary referral-based hospitals, Severance Hospital and Gangnam Severance Hospital, affiliated with Yonsei University Health System (YUHS). Consecutive patients who visited the departments of Dermatology and Ophthalmology between June 2007 and May 2017 who were histologically diagnosed as SC, sebaceous adenoma and sebaceoma were included and their medical records from the electronic health record (EHR) system of YUMC were reviewed.

\section{Analysis of clinical data}

Patients were divided into two groups: the SC group and the BSN group, which included sebaceous adenomas and sebaceomas. We compared the basic clinical data obtained for all patients between the two groups including age, sex, duration, location of the primary tumor, tumor size, comorbidity, treatment, recurrence and presence of distant metastases. Among the patients with presence of associated visceral malignancies, we obtained and compared further additional clinical data including the age at diagnosis of visceral malignancies and the number of associated visceral malignancies. We received the patient's consent form about publishing all photographic materials.

\section{Statistical analysis}

The SC group and the BSN group were compared statistically. Statistical analyses were performed using SPSS software ver. 23.0 (IBM Co., Armonk, NY, USA). The results were expressed as means \pm standard deviations. The unpaired t-test was performed for continuous variables. To compare the categorical data, the chi-square test or the Fisher exact test was used. $p$-value $<0.05$ were considered to be statistically significant.

\section{Ethics statement}

This study was reviewed and approved by the Institutional Review Board of the Gangnam Severance Hospital, Yonsei University College of Medicine, Seoul, Korea (IRB no. 3-2018-0014) and adhered to the tenets of the Declaration of Helsinki.

\section{RESULTS}

\section{Patient demographics}

A total of 80 patients with sebaceous neoplasms were included in this study. Of the 80 patients, 51 were diagnosed with SC and 29 with BSN. The detailed demographic and clinical characteristics are provided in Table 1. The mean age at diagnosis was $62.6 \pm 15.6$ (range, 36 94) years for patients with SCs and $58.4 \pm 14.7$ (range, $31 \sim$ 87 ) years for patients with BSNs. The mean age at onset of the sebaceous neoplasms was $60.0 \pm 18.7$ (range, 19 92) years for SC patients and $50.9 \pm 18.6$ (range, $10 \sim 87$ ) years for BSN patients. The mean age at onset was earlier in the BSN group than the SC group. In terms of distribution of sex, SCs had a female dominant distribution (M:F=1:1.8), whereas BSNs did not show gender differences. The most frequent location of SCs and BSNs was the head and neck area $(49 / 51,96.1 \%$ and 28/29, 96.6\% respectively). SCs were most frequently located on the eyelids (41/51, $80.4 \%$ ), while BSNs did not showed any site predilection. In the SC group, 50 patients showed solitary lesions and just one patient showed multiple lesions. In the BSN group, 4 of 29 patients had multiple lesions. There was no difference in the mean size of tumor at diagnosis $(\mathrm{SC}, 1.4 \mathrm{~cm}$; $\mathrm{BSN}, 1.1 \mathrm{~cm})$. Clinical and histopathologic presentations of sebaceous neoplasms are shown in Fig. 1, 2. In the SC group, hypertension $(11 / 51,21.6 \%)$ was the most frequently associated comorbidity followed by diabetes mellitus (9/51, 17.7\%). Associated visceral malignancy was observed in 8 patients (15.7\%). In the BSN group, visceral malignancy was the most frequently associated comorbidity $(6 / 29,20.7 \%)$, followed by hypertension $(5 / 29$, $17.2 \%)$.

\section{Treatment and prognosis}

For the treatment of SCs, 6 patients $(11.8 \%)$ received Mohs micrographic surgery (MMS) and 44 (86.2\%) received wide local excision (WLE). The final 1 patient received radiation therapy due to old age and a poor general condition. In the BSN group, 2 patients $(6.9 \%)$ underwent MMS and 15 (51.7\%) underwent WLE. Five patients $(17.2 \%)$ received cryotherapy or laser therapy and 7 $(24.1 \%)$ did not receive any treatment. The mean follow- 
Table 1. Baseline clinical characteristics of 80 sebaceous neoplasm patients

\begin{tabular}{|c|c|c|}
\hline Factors & $\begin{array}{c}\text { Sebaceous } \\
\text { carcinoma } \\
(n=51)\end{array}$ & $\begin{array}{c}\text { Benign } \\
\text { sebaceous } \\
\text { neoplasms } \\
(\mathrm{n}=29)\end{array}$ \\
\hline Age at diagnosis (yr) & $62.6 \pm 15.6$ & $58.4 \pm 14.7$ \\
\hline \multicolumn{3}{|l|}{ Gender } \\
\hline Male & $18(35.3)$ & $14(48.3)$ \\
\hline Female & $33(64.7)$ & $15(51.7)$ \\
\hline Duration (mo) & $23.7 \pm 39.4$ & $85.7 \pm 148.5$ \\
\hline Age at onset of SN (yr) & $60.0 \pm 16.6$ & $50.9 \pm 18.6$ \\
\hline \multicolumn{3}{|l|}{ Location } \\
\hline Head and neck & $49(96.1)$ & 28 (96.6) \\
\hline Scalp & $2(3.9)$ & $6(20.7)$ \\
\hline Forehead & $1(2.0)$ & $1(3.5)$ \\
\hline Temple & $0(0.0)$ & $3(10.3)$ \\
\hline Eyelid & $41(80.4)$ & $3(10.3)$ \\
\hline Ear & $2(4.0)$ & $2(6.9)$ \\
\hline Nose & $1(2.0)$ & $4(13.8)$ \\
\hline Cheek & $1(2.0)$ & $7(24.1)$ \\
\hline Philtrum & $1(2.0)$ & $0(0.0)$ \\
\hline Neck & $0(0.0)$ & $2(6.9)$ \\
\hline Trunk & $2(3.9)$ & $1(3.5)$ \\
\hline Size $(\mathrm{cm})$ & $1.4 \pm 1.3$ & $1.1 \pm 0.8$ \\
\hline \multicolumn{3}{|l|}{ Comorbidity } \\
\hline Hypertension & $11(21.6)$ & $5(17.2)$ \\
\hline Diabetes mellitus & $9(17.7)$ & $3(10.3)$ \\
\hline $\begin{array}{l}\text { Coronary artery } \\
\text { obstructive disease }\end{array}$ & $3(5.9)$ & $1(3.5)$ \\
\hline $\begin{array}{l}\text { Benign prostatic } \\
\text { hyperplasia }\end{array}$ & $1(2.0)$ & $3(10.3)$ \\
\hline Tubular adenoma & $1(2.0)$ & $4(13.8)$ \\
\hline Visceral malignancy & $8(15.7)$ & $6(20.7)$ \\
\hline \multicolumn{3}{|l|}{ Treatment } \\
\hline $\begin{array}{l}\text { Mohs micrographic } \\
\text { surgery }\end{array}$ & $6(11.8)$ & $2(6.9)$ \\
\hline Wide local excision & $44(86.2)$ & $15(51.7)$ \\
\hline Radiotherapy & $1(2.0)$ & $0(0.0)$ \\
\hline Cryotherapy & $0(0.0)$ & $2(6.9)$ \\
\hline Laser & $0(0.0)$ & $3(10.3)$ \\
\hline None & $0(0.0)$ & $7(24.1)$ \\
\hline Recurrence & $4(7.7)$ & $1(3.5)$ \\
\hline Local recurrence & $1(2.0)$ & $1(2.0)$ \\
\hline Distant metastases & $3(5.9)$ & $0(0.0)$ \\
\hline Follow-up period (mo) & $27.9 \pm 26.3$ & $18.3 \pm 34.0$ \\
\hline
\end{tabular}

Values are presented as mean \pm standard deviation or number (\%). SN: sebaceous neoplasm.

up period was $27.9 \pm 26.3$ months for SC patients and $18.3 \pm 34.0$ months for BSN patients and there was no significant difference between the two groups. During the follow-up period, recurrence was observed in 4 SC patients: 1 had a local recurrence while the other 3 patients showed distant metastasis to the lung, parotid gland and neck lymph nodes. The mean duration to recurrence was $13.25 \pm 5.6$ (range, $7 \sim 20$ ) months. Among the BSN group, 1 patient who was treated with ablative $\mathrm{CO} 2$ laser showed local recurrence.

\section{Sebaceous neoplasms and visceral malignancies}

The clinical data of patients with associated visceral malignancies are shown in Table 2 and Table 3. A total of 14 patients $(17.5 \%)$ had associated visceral malignancy; $8 / 51$ patients $(15.7 \%)$ were in the SC group and 6/29 (20.7\%) with in the BSN. In the SC group, patients were found to have only one visceral malignancy. Interestingly, among the 6 patients with BSNs, 3/6 patients (50.0\%) were associated with multiple visceral malignancies, while the other 3 patients had 1 visceral malignancy: among those with multiple malignancies, 1 patient had 3 and 2 patients were found to have 2 visceral malignancies. Over all, in this subset of patients with confirmed visceral malignancy, the mean age at diagnosis of the sebaceous neoplasms was $68.1 \pm 10.9$ (range, 46 82) years for SC patients and $53.7 \pm 4.8$ (range, $46 \sim 59$ ) years for $\mathrm{BSN}$ patients. The mean age at diagnosis of the visceral malignancies was $63.9 \pm 10.3$ (range, $47 \sim 79$ ) years for SC patients and $47.5 \pm 5.7$ (range, $41 \sim 55$ ) years for BSN patients. The diagnosis of sebaceous neoplasms preceded that of visceral malignancy by 4.2 and 6.4 years in SC and BSN patients, respectively. Two patients with SC were diagnosed with a subsequent visceral malignancy during the process of work-up.

A total of 18 visceral malignancies were found in 14 patients, 8 in the SC group and 10 in the BSN group. The detailed characteristics of the associated visceral malignancies can be seen in Table 4. Among the 18 visceral malignancies, the most common site was the gastrointestinal (Gl) tract $(9 / 18,50.0 \%)$. In particular, we found $4 \mathrm{Gl}$ tract cancers that were associated with $\mathrm{BSN}$ and all were diagnosed as colon or rectal cancers. The other sites involved were the bile duct, thyroid, breast, ureter, prostate and uterus. The histologic type of visceral malignancies was described in 13 visceral malignancies, and all were adenocarcinoma (13/18, 72.2\%).

\section{DISCUSSION}

Sebaceous neoplasms are widely classified into 3 types of tumors; SC, sebaceous adenomas and sebaceomas. SC is a rare malignant neoplasm of the sebaceous glands, commonly diagnosed in older adults and with no known gender predilections ${ }^{3}$. SC is divided into extraocular and ocular types according to the location of the tumor. The ocular type, which is located on the periorbital area, account 


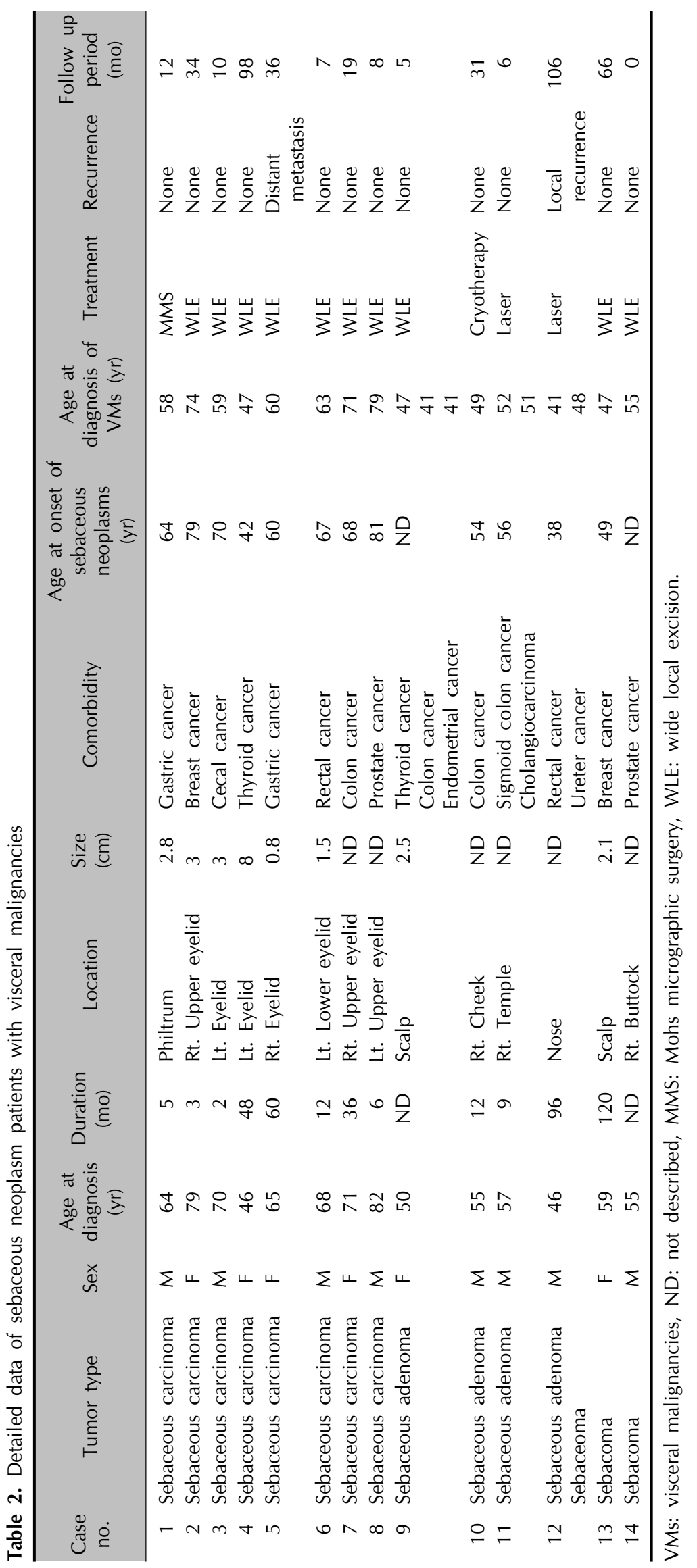



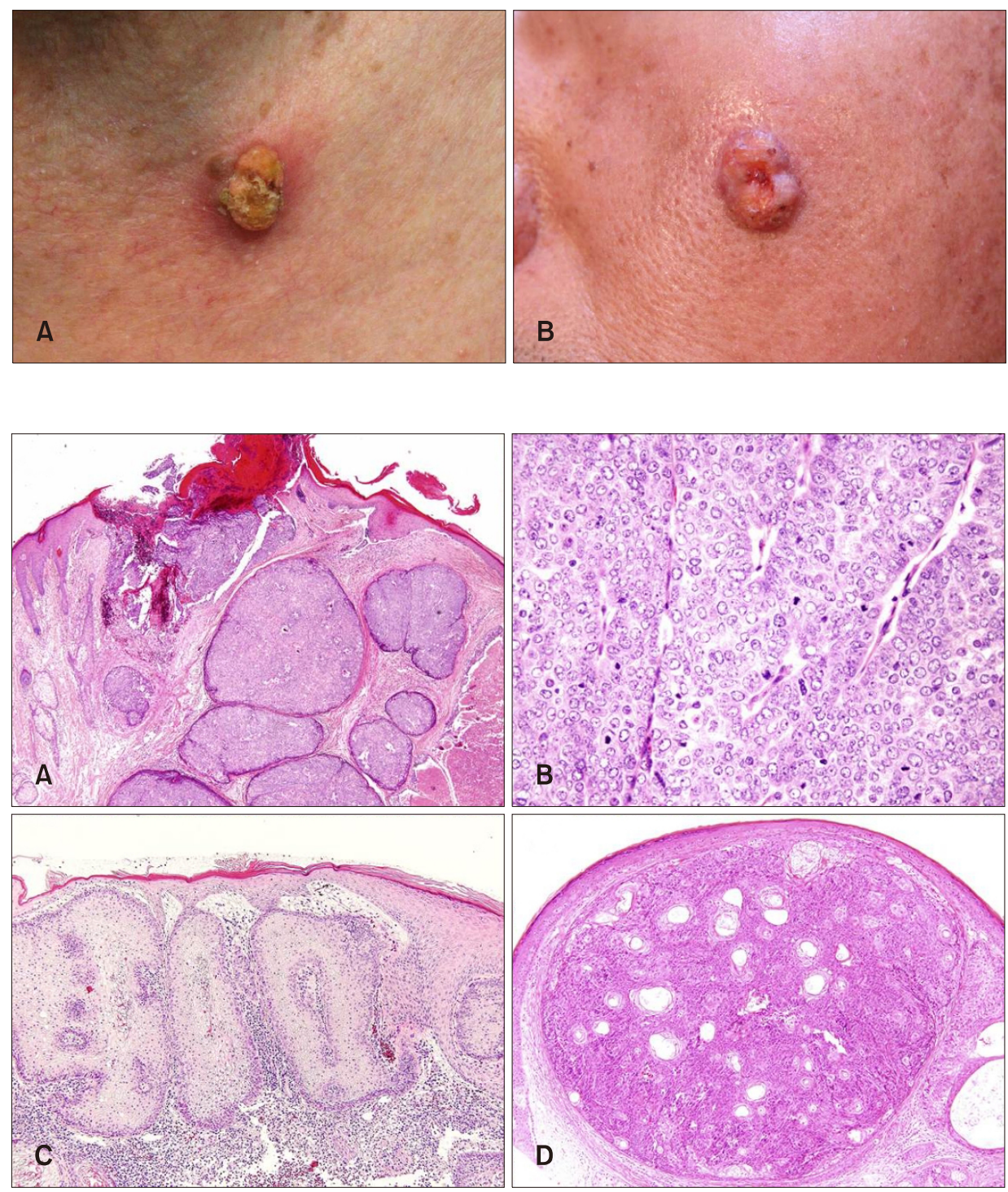

Fig. 1. Clinical presentations of sebaceous neoplasms. (A) Extraocular sebaceous carcinoma presenting as a crusted yellowish nodule with erythematous base on the neck. (B) Sebaceous adenoma presenting as an erythematous nodule with central umbilication on the left cheek.

Fig. 2. Histopathologic presentations of sebaceous neoplasms. (A, B) Sebaceous carcinoma. (A) Multilobular basaloid tumors are associated with epidermal ulceration (H\&E, $\times 40$ ). (B) The constituent cells, both basaloid cells and sebocytes, demonstrate marked cytologic atypia and mitotic activity $(\mathrm{H} \& \mathrm{E}, \times 400)$. (C, D) Benign sebaceous neoplasms. The tumor is composed of enlarged sebaceous lobules with two types of mature cells: sebaceous and basaloid cells $(H \& E, \times 100)$. (C) Sebaceous cells predominate in sebaceous adenoma and (D) basaloid cells in sebaceoma $(H \& E, \times 100)$. for $75 \%$ of $\mathrm{SC}^{3}$. Typically, SC presents as a pink or yellow nodule or plaque. BSN includes sebaceous adenoma and sebaceoma. Sebaceous adenomas typically presents as solitary or multiple nodules less than $1 \mathrm{~cm}$ in size with tan, pink or yellow coloring. The clinical presentation of sebaceoma is not distinct, with appearance of papular, nodular, keratotic or ulcerated lesions. These two neoplasms are distinguished with histological features. Sebaceoma is diagnosed when it is composed with greater than $50 \%$ of germinative basaloid cells. Otherwise the diagnosis of sebaceous adenoma can be made ${ }^{3}$. In this study, we sought to assess the clinical features of patients with SC and BSN. We also evaluated the incidence and clinical characteristics of associated visceral malignancies. In our study, the mean age at diagnosis was 62.6 years, which was at a younger age compared with previously published studies. Additionally, our results showed a fe- male predilection $(64.7 \%)$. The ocular type occupied $80.4 \%$ of the total SC, which was similar to previously published data. The differences in these results may be due to several factors. First, our data is based on the Korean population. According to Park et al.'s report ${ }^{4}$, which analyzed the clinicopathologic data of Korean SC patients, the mean age of ocular SC was found to be 69.19 and extraocular SC was 67.46 which was also younger than other previously published studies based of other ethnic populations. This study also revealed a slight female predilection. Second, as SC is a rare tumor with a prevalence of 1 per 1,000,000, the total number of subjects and small differences may not be large enough to effectively power a statistically significant comparison. However, while further studies with larger number of subjects and multivariate analysis are needed, we believe our study is still meaningful and contributes significantly as there are 
Table 3. Comparison of clinical features regarding visceral malignancies between patients with sebaceous carcinoma and benign sebaceous neoplasm

\begin{tabular}{lcc}
\hline \multicolumn{1}{c}{ Factors } & $\begin{array}{c}\text { Sebaceous } \\
\text { carcinoma } \\
(\mathrm{n}=8)\end{array}$ & $\begin{array}{c}\text { Benign sebaceous } \\
\text { neoplasms } \\
(\mathrm{n}=6)\end{array}$ \\
\hline $\begin{array}{l}\text { Age at diagnosis of } \\
\text { sebaceous neoplasms (yr) }\end{array}$ & $68.1 \pm 10.9$ & $53.7 \pm 4.8$ \\
$\begin{array}{l}\text { Gender } \\
\text { Male }\end{array}$ & $4(50.0)$ & $4(66.7)$ \\
$\quad$ Female & $4(50.0)$ & $2(33.3)$ \\
$\begin{array}{l}\text { Number of visceral } \\
\text { malignancies }\end{array}$ & & \\
1 & $8(100.0)$ & $3(50.0)$ \\
2 & $0(0.0)$ & $2(33.3)$ \\
3 & $0(0.0)$ & $1(16.7)$ \\
Age at onset of sebaceous & $66.4 \pm 12.1$ & $49.3+13.5$ \\
neoplasms (yr) & $63.9 \pm 10.3$ & $47.5 \pm 5.7$ \\
Age at diagnosis of visceral \\
malignancies (yr)
\end{tabular}

Values are presented as mean \pm standard deviation or number $(\%)$.

not enough previously published statistical data regarding $\mathrm{SC}$ in Korean patients.

There is little currently known fact regarding BSN, with insufficient demographic data concerning BSN separatedly from SC. In our study, the mean age at diagnosis of BSN was 58.4 years and there were no gender predilections. The most common location of the tumors was the head and neck $(96.6 \%)$, but only 3 cases $(10.3 \%)$ occurred in the ocular area. Overall, we found no significant differences between the SC group and the BSN group except for the location of the tumors.

While the standard treatment for SC is WLE, recently, MMS has emerged as an adequate alternative treatment. Considering that location of SC is most frequently in cosmetically sensitive areas such as the eyelids and face, MMS would be advantageous because of its superior tumor margin control and preservation of normal skin. Furthermore, there are studies reporting that MMS has a lower local recurrence rate than $\mathrm{WLE}^{5-7}$. We also consider WLE and MMS as first line treatments in our medical centers, and therefore in our study, surgical excision was the first line treatment except for one patient who was unable to undergo surgery. Among the 44 patients who were treated with WLE, only one patient showed local recurrence. There was no local recurrence in patients $(n=6)$ who were treated with MMS.

MTS is a rare autosomal-dominant disorder characterized by the association of sebaceous gland tumors or other skin tumors with one or multiple visceral malignancies. The most commonly associated skin neoplasm is sebaceous
Table 4. Type of visceral malignancies associated in sebaceous neoplasm patients

\begin{tabular}{lll}
\hline \multicolumn{1}{c}{ Factors } & $\begin{array}{c}\text { Sebaceous } \\
\text { carcinoma } \\
(\mathrm{n}=8)\end{array}$ & $\begin{array}{c}\text { Benign } \\
\text { sebaceous } \\
\text { neoplasms } \\
(\mathrm{n}=10)\end{array}$ \\
\hline $\begin{array}{l}\text { Location of visceral } \\
\text { malignancies }\end{array}$ & & \\
Gl tract & $5(62.5)$ & $4(40.0)$ \\
$\quad 2(25.0)$ & $0(0.0)$ \\
$\quad$ Comach & $2(25.0)$ & $3(30.0)$ \\
$\quad 1(12.5)$ & $1(10.0)$ \\
Rectus & $0(0.0)$ & $1(10.0)$ \\
Thyroid & $1(12.5)$ & $1(10.0)$ \\
Breast & $1(12.5)$ & $1(10.0)$ \\
Ureter & $0(0.0)$ & $1(10.0)$ \\
Prostate & $1(12.5)$ & $1(10.0)$ \\
Uterus & $0(0.0)$ & $1(10.0)$ \\
Histologic type of visceral & & \\
malignancies & $7(87.5)$ & $6(60.0)$ \\
Adenocarcinoma & $1(12.5)$ & $4(40.0)$ \\
Not described &
\end{tabular}

Values are presented as number (\%). Gl: gastrointestinal.

adenoma with a frequency of $68 \%$, followed by SC and sebaceoma ${ }^{8}$. The most commonly associated visceral malignancy is colorectal adenocarcinoma. The diagnosis of MTS can be confirmed by immunohistochemistry on lesions which shows loss of staining for MMR gene products with high sensitivity ${ }^{8}$.

However, detailed clinical data is sparse with few reports including the MTS portion of all patients with sebaceous neoplasms. Such information would be valuable for clinicians in guiding decisions on whether further evaluation for MTS should be required in patients with sebaceous neoplasm.

In our study, 14 patients (17.5\%) were found to have associated visceral malignancies: 8 patients $(15.7 \%)$ in the SC group and 6 patients $(20.7 \%)$ in the BSN group. The proportion of patients associated with visceral malignancies is not statistically significant but is greater in the BSN group than in the SC group. Interestingly, SC group was also highly associated with visceral malignancy despite the lower incidence of SC associated with MTS compared with $\mathrm{BSN}^{8}$. Among these patients, 3 patients in the BSN group $(50.0 \%)$ had multiple visceral malignancies. Among the 18 visceral malignancies noted, 50\% were of Gl tract origin. According to the Korean National Cancer Center, the total cancer prevalence rate was $2.9 \%$, with $0.6 \%$ and $0.5 \%$ prevalence rates in gastric cancers and colorectal cancers, respectively ${ }^{9}$. Compared to this data, the patients in our study showed meaningfully high incidence rates of 
visceral malignancies, especially for Gl tract cancers. However, patients with visceral malignancy would have had regular follow-up on outpatient clinic, and those with skin lesions would have been referred to the department of dermatology. This could be one of the reasons for a higher incidence of visceral malignancy in patients with sebaceous neoplasm. Additionally, in the BSN group, the mean age at diagnosis of visceral malignancy was 47.5 years; for the colorectal cancers, the mean age was 45.8 years. According to the American Cancer Society, colorectal cancer is commonly diagnosed after the age of 50 in the majority of people, with a similar tendency noted in Asian ethnic groups ${ }^{10}$. When we compare these data, we note that the patients in our study developed visceral malignancies at a much younger age. We also checked the order of the incidence of sebaceous neoplasms and visceral malignancies in 14 patients. Although a distinct relationship was not found, the diagnosis of sebaceous neoplasms preceded that of visceral malignancy by 4.2 and 6.4 years in average in SC and BSN, respectively. In addition, among the 14 patients, 2 patients with SC had subsequently discovered visceral malignancy during the process of work-up for surgery of SC. These results suggest that screening for visceral malignancy, especially for the Gl tract cancers, may be beneficial for patients with sebaceous neoplasms. Based on our results, we cautiously recommend immunohistochemistry for MMR gene products $^{8,11}$, and additionally, esophagogastroduodenoscopy or colonoscopy could be recommended for the patients with sebaceous neoplasms ${ }^{12}$. Considering that sebaceous neoplasms are visible lesions which can be easily diagnosed with skin biopsy, it would be efficient if this process contribute to finding visceral malignancies.

This study has several limitations. First, it was a retrospective study with inherent limitations. Second, its study population was limited to Korean patients in a tertiary university hospital setting. Third, the number of subjects is small and may not effectively power statistical analysis for significance. Nevertheless, the strengths of this study are that it provides information regarding the clinical characteristics of previously little-known sebaceous neoplasms and associated visceral malignancies. In particular, this study suggests the necessity of screening for visceral malignancy, especially of the Gl tract, in patients with sebaceous neoplasms.

Although further studies with a big sample sizes and additional studies about the efficacy of the screening for the visceral malignancies would be needed, we expect this study to contribute to comprehending and working-up of sebaceous neoplasms.

\section{ACKNOWLEDGMENT}

This research was supported by the Basic Science Research Program through the National Research Foundation of Korea (NRF) funded by the Ministry of Science, ICT \& Future Planning (2017R1C1B2005574).

\section{CONFLICTS OF INTEREST}

The authors have nothing to disclose.

\section{ORCID}

Jee Eun Kim, https://orcid.org/0000-0003-0795-5742

Jong Hoon Kim, https://orcid.org/0000-0002-3385-8180

Kee-Yang Chung, https://orcid.org/0000-0003-3257-0297

Jin Sook Yoon, https://orcid.org/0000-0002-8751-9467

Mi Ryung Roh, https://orcid.org/0000-0002-6285-2490

\section{REFERENCES}

1. Moscarella E, Argenziano G, Longo C, Cota C, Ardigò M, Stigliano $\mathrm{V}$, et al. Clinical, dermoscopic and reflectance confocal microscopy features of sebaceous neoplasms in Muir-Torre syndrome. J Eur Acad Dermatol Venereol 2013; 27:699-705.

2. Flux K. Sebaceous neoplasms. Surg Pathol Clin 2017;10: 367-382.

3. lacobelli J, Harvey NT, Wood BA. Sebaceous lesions of the skin. Pathology 2017;49:688-697.

4. Park SK, Park J, Kim HU, Yun SK. Sebaceous carcinoma: clinicopathologic analysis of 29 cases in a tertiary hospital in Korea. J Korean Med Sci 2017;32:1351-1359.

5. Snow SN, Larson PO, Lucarelli MJ, Lemke BN, Madjar DD. Sebaceous carcinoma of the eyelids treated by Mohs micrographic surgery: report of nine cases with review of the literature. Dermatol Surg 2002;28:623-631.

6. Spencer JM, Nossa R, Tse DT, Sequeira M. Sebaceous carcinoma of the eyelid treated with Mohs micrographic surgery. J Am Acad Dermatol 2001;44:1004-1009.

7. Harvey DT, Taylor RS, Itani KM, Loewinger RJ. Mohs micrographic surgery of the eyelid: an overview of anatomy, pathophysiology, and reconstruction options. Dermatol Surg 2013;39:673-697.

8. John AM, Schwartz RA. Muir-Torre syndrome (MTS): an update and approach to diagnosis and management. J Am Acad Dermatol 2016;74:558-566.

9. Jung KW, Won YJ, Oh CM, Kong $\mathrm{HJ}$, Lee $\mathrm{DH}$, Lee $\mathrm{KH}$; Community of Population-Based Regional Cancer Registries. Cancer statistics in Korea: incidence, mortality, survival, and prevalence in 2014. Cancer Res Treat 2017;49:292-305.

10. Siegel RL, Miller KD, Fedewa SA, Ahnen DJ, Meester RGS, Barzi A, et al. Colorectal cancer statistics, 2017. CA Cancer J Clin 2017;67:177-193. 
11. Oliveira CM, Campos JG, Maia MR, Lobo LE, Valle FF. The role of immunohistochemistry in the Muir-Torre syndrome. An Bras Dermatol 2015;90(3 Suppl 1):168-170.
12. Ponti G, Ponz de Leon M. Muir-Torre syndrome. Lancet Oncol 2005;6:980-987. 